\title{
A new weapon to boost cancer immunotherapy
}

In recent years,

immunooncology has paved new avenues for effective

treatment across cancer

types. In addition to cytotoxic

harness the power of the

human immune respons

against malignant cells.

However, immunotherapy is not

perfect, and as always, cancer

has mechanisms to evade

these attacks. Dr Svetlana

Hamm, Head of Research and

in Germany, has uncovered

in Gexiting, has uncovered

therapy which has the ability to

increase anti-tumour efficacy

of immunotherapies. 4SC, a

German biotech company, is

developing this combinatio

for treatment of cancer

patients resistant to $P D-1 /$
$P D-L 1$ therapy.
In the last few years, immunotherapy has become one of the leading methods stimulates the immune system to destroy tumours more effectively and can be used in monotherapy as well as in combination with other cancer therapies or surgen. Now, scientists are finding ways to refine immunotherapy through enhancing the tumour cell immunogenicity, increasing the number of immune cells at the tum site, or by blocking specific molecules which cancer cells produce to supress the immune system's attack on the tumour.

One particularly exciting immunotherapy combination approach has been discovered by the Research and holational headed by Dr Svetlana Hamm. Along with her team, she is exploring the potential of called domatinostat Domatinostat works in conjunction with immunotherapy, specifically one that targets
1 (programmed death protein 1 and PD

\section{ANCER IMMUNITY AND}

THE PD-1/PD-L1 AXIS

cells can result in a

dysregulation of cellular processes and

uncontrolled cell proliferation, eventually

causing tumourigenesis. On the other

hand, mutations and dysregulated

gene expression cause tumour cells to

produce tumour antigens and release

gnals, marking them as foreign and

dangerous to the organism. Depending

on how visible this process is to the

and, he inm nune system will respond

cancer cells such cells. However, often block the immune attack.

The immune response generally involves an influx of specific cells of the immune system, so-called cytotoxic T cells specifically kill tumour cells through recognition of their tumour antigens However, tumour cells can block this attack by upregulating the PD-L1 molecule on the surface. PD-L1 binds to the inhibitory PD-1 receptor expressed on CTLS, blocking their activity and rendering them essentially useless. The so-called checkpoint blockade is one form of immunotherapy which stops the PD-L1 molecule from binding to the CTL's PDreceptor, thereby unleashing the CTL atlack on the tumour cells. As successful as this approach has been in many between different cancer types greaty
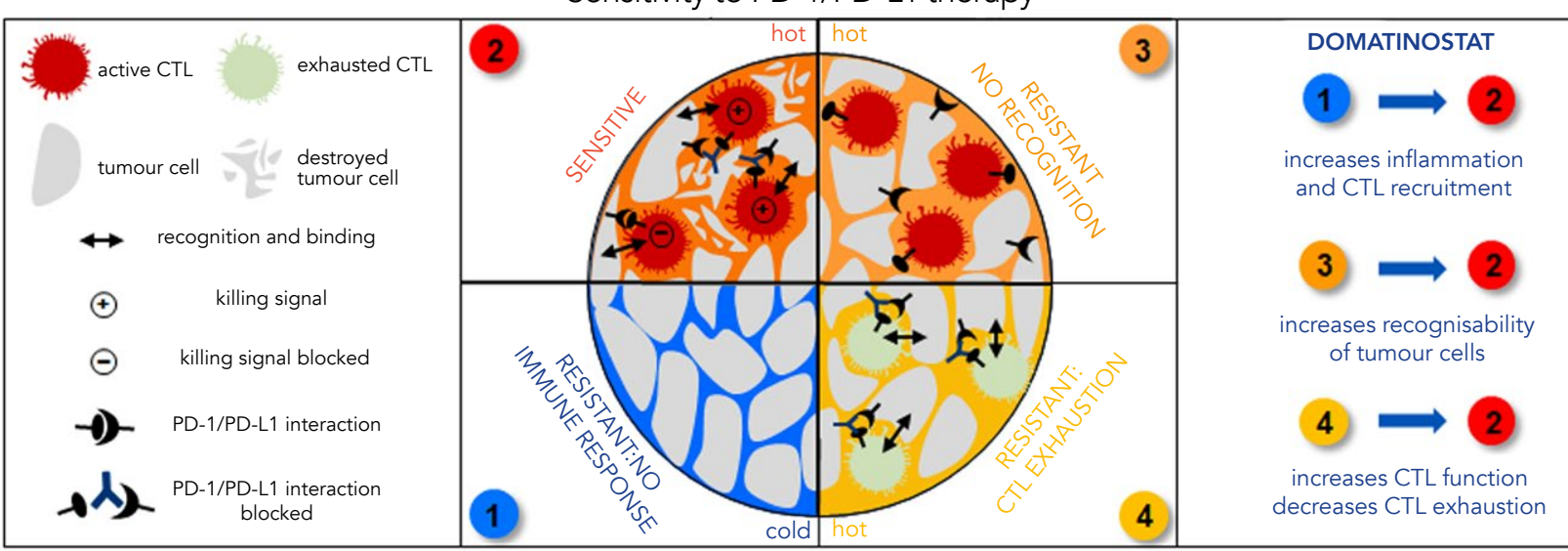

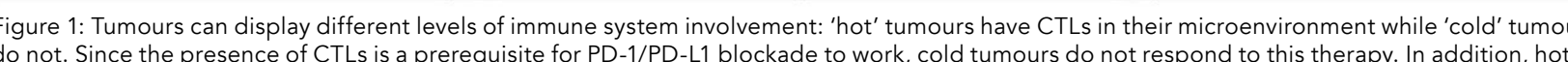
tumours can also become resistant to PD-1/PD-L1 blockade by avoiding detection or by pushing the CTLs into an exhausted state.

between individua patents. This can be elue to the absence of a This can be due to the absence of a tumour-specific immune response, downregulation of immurspecific assive cells and molon, or produsupt bs the cencer cells to inhibit CTL function, independently of the

of PD-L1.

CANCER CELLS HAVE MANY DEFENCE MECHANISMS

Tumours can display different levels of immune system involvement. 'Hot' tumours have CTLs present in the tumour immune microenvironment but are actively expressing PD-L1 as a defence to suppress the CTL attack (see figure 1, panels 2, 3, and 4). 'Cold' tumours do not have inmune cells in ent and likely have avoided immune detection, so there is no need for these
tumour cells to upregulate PD-L1 (panel 1). In this case, the aim of the therapy is to 'heat up' the tumour and cause CTLs to infiltrate. Checkpoint inhibitors the microenvironment still manages to inhibit the effectivity of CTLs. The CTLs are then in a so-called exhausted The fore, a drug is needed tat work ale, a dis is coded that can checkpoint therapy.

HDAC inhibitors are a group of synthetic molecules which have been shown to increase the expression of cancer antigens and their presentation to CTLS, as well as the number of CTLs in immune cells to the tumour.

Domatinostat, which is a specific HDAC

varying CTL levels ' 'hot' or 'cold' tumours). The mice were then treated homath dostat and PD-1/PD-L1 established human checkpoint inhibitors like nivolumab, pembrolizumab, or avelumab). CT26 tumours are a type of tumour that is considered noninflamed (cold) and C38 tumours are inflamed (hot)

The results were clear. In the cold CT26 tumours, domatinostat alone decreased the tumour size by half and increased the number of CTLs by eightfold (see figure 2). Furthermore, the treatment triggered the immune-related cellular machinery, enhancing inflammation and turning the tumour into a hot one. Domatinostat
also increased the recognisability of

Domatinostat aims to restart the immune system's attack on the tumour and works in conjunction with immunotherapy, specifically one that

targets the PD-1/PD-L1 axis. CTLs by upregulating the production of their presentation on the cell surface. When a combinatio of domatinostat dministered, the the tumour secific immune response by blocking the PD-1/PD-L1 interaction, resulting in the killing of cancer cells (panel 2).

Unfortunately, tumours fight back. Tumour cells try to avoid recognition and binding by CTLs through downregulation of antigen presentation (panel 3), or they inhibit CTL function As a result, which where PD-1/PD-11 is sucesstily bocked, monotherapy. Given the varied effectivity of PD-1/PD-L1 blockade dependent on the microenvironment, Dr Hamm and her team at 4SC proposed that the addition of domatinostat would combat cancer defences causing resistance to checkpoint therapy

TESTING DOMATINOSTAT IN TUMOUR-BEARING MICE The firs step was to tes domatinos administered, the
anti-tumoural effect was increased further, whereas PD-1/PD-L1 blockers alone were not effective.

The results from the hot C38 tumour model were similarly impressive. Although C38 tumours already accommodate a high number of CTLs, these cells are not able to eliminate the tumours because of her exhausted /

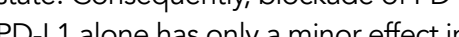




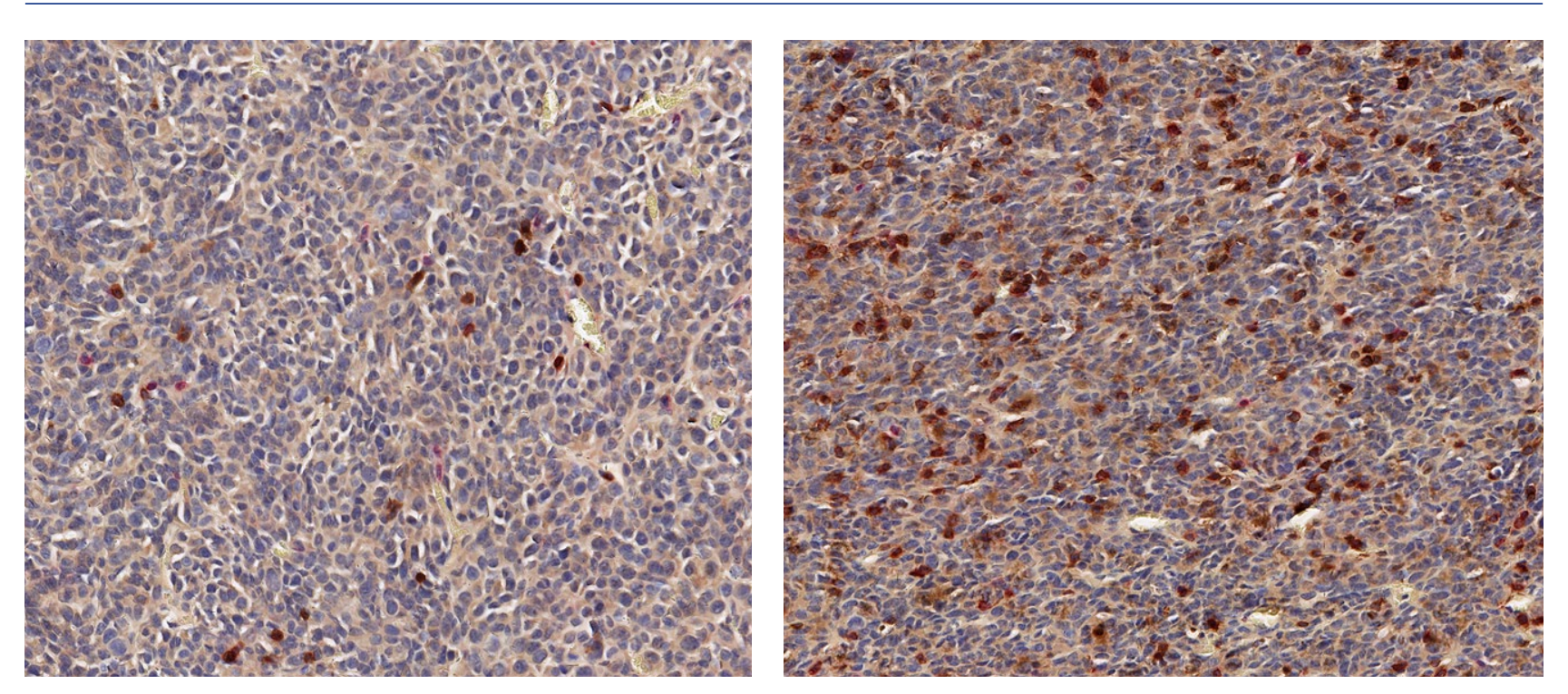

Figure 2: The infiltration of CTLs into the tumour can be analysed by staining these cells with specific colours. Here, tumour cells are blue and CTLS
are brown. Domatinostat strongly increases the number of CTLs in the tumour (on the right) compared to the untreated tumours (on the left). this type of cancer.
Domatinostat,

Domatinost

managed to

increase the

number of CTLS

in these tumours

by nearly twofold

and more and effectivity. As a result, a greatly increased survival time was achieved, both with domatinostat alone as well as in combination with PD-1/PD-Li blockade, which resulted in complete healing of more than $50 \%$ of the test animals.

\section{ADDING TO THE COCKTAIL} MHC-II (major histocompatibility complex-ll) molecules are proteins tumour-specific CTL types, thereby enhancing the immune attack on the to increase MHC-II molecules in tumours. However, this beneficial effect on the immune response generation is often found to be levelled out by a negative effect on CTL activity due to the binding of MHC-II to another inhibitory CT receptor LAG3, which blocks the function experiment with tumour-bearing mice, Dr Hamm and her team at 4SC added a LAG3 blocker to the mix of domatinosta the tumour reg.
animals tested. support the generation of adlitional tumour-Domatinostat has been shown of CTLs similarly to PD-1. Thus, in anoth and PD-1/PD-L1 blockade. This resulted in even higher anti-tumour activity, wi relevant genes in the tumours of patients before and
after administration after administration They observed increased expression
of cancer antigens, antigen presenting machinery and enhanced inflammation in the first patients evaluated, which confirms the observations from the prior animal tests, and implies an increased susceptibility to the subsequent treatment with checkpoint inhibitors.

FROM BENCH TO BEDSIDE Domatinostat has proved itself in these early tests to be a very PD-1/PD-L1 inhibitors As der for above, it accomplishes this by not only increasing CTL count in the tumour micreenvironment but also by enhancing CTL activity and pathways which combat the cancer cell resistance mechanisms. These effects are expected to be particularly usefu in cancers like melanoma, Merkel cell carcinoma (MCC), urothelial cancer, and colorectal carcinoma.

These early biomarker results for domatinostat therapy from the the whole $45 \mathrm{C}$ team is looking forward to continuing development of this increased expression effectivity, and activating immune SENSTIZE trial are very encouraging an potentially practise-changing drug for

\section{Dr Svetlana Hamm}

\section{Behind the Research}

\section{Research Objectives}

Dr Hamm and her team are examining the HDAC interes.

\section{Detail}

Bio

Dr Svetlana Hamm has been working in the area of cancer immunology formore than 15 years. She is developing new therapies supporting the patient's immune system with the aim to change the way cancers will be treated in future, providing lasting responses and definitive cure.

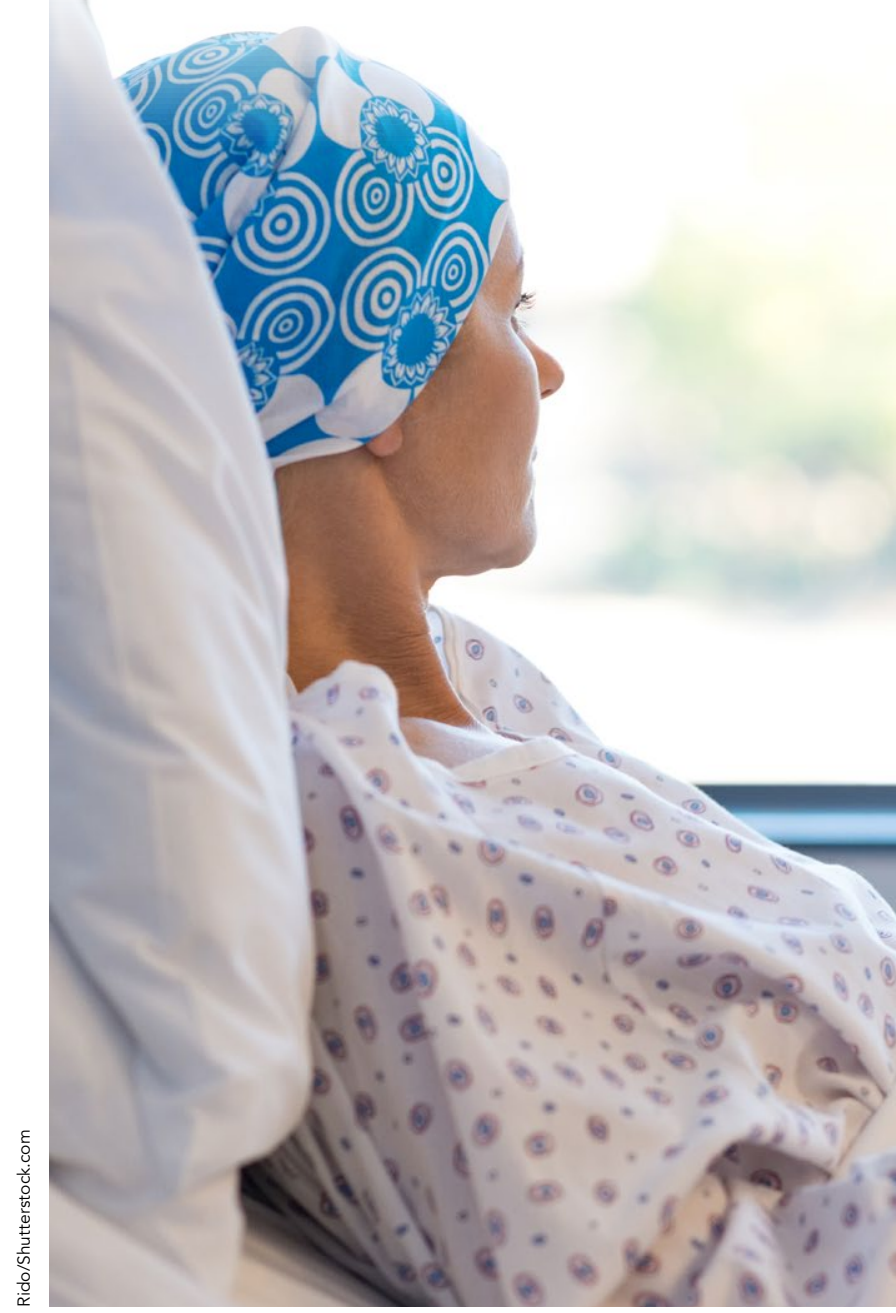

\section{References}

Bretz, A. et al. (2019). Domatinostat favors immunotherapy by modulating TIME. Journal for ImmunoTherapy of Cancer, 7, 294. Available at: https://doi.org/10.1186/ s40425-019-0745-3

Hassel, J.C. et al. (2019). Phase Ib/ll study (SENSITIZE) assessing safety, pharmacokinetics (PK), pharmacodynamics (PD), and clinical outcome of domatinostat in combination with pembrolizumab non-responding to prior checkpoint inhibitor Annals of Oncology 30 v559. Available at. https.//doi. org/10.1093/annonc/mdz255.058. Poster available https://wwww 4sc de/wp-content/uploads/SENSITIZE Poster ESMO final.pdf

Hegde, P.S. et al. (2016). The Where, the When, and the How of Immune Monitoring for Cancer Immunotherapies in the Era of Checkpoint Inhibition. Clinical Cancer Research, 22, 1865-74. Available at: https://clincancerres aacrjournals.org/content/22/8/1865

\section{Personal Response}

What did the toxicity profile look like in the patients

II Domatinostat alone, as well as in combination with pembrolizumab, was safe and well-tolerated in patients. events have been observed and the SENSITIZE trial continues to recruit patients. Additionally, domatinostat is under evaluation in further clinical trials testing the combination of domatinostat with checkpoint inhibitors in patients with colorectal and gastric cancer or with

inhibitor therapy.
in

The next step was to test domatinostat is an ongoing clinical patientswith melanoma who the ideal se cup fockpoint blockade alone et al., 2019).

In this trial, domatinostat is given as monotherapy for two weeks before 4SC team monitors the expression
II 